\title{
Rate-based path selection based on link metric optimization in SPBM
}

\author{
Yu Nakayama ${ }^{\text {a) }}$ and Ryoma Yasunaga \\ NTT Access Network Service Systems Laboratories, NTT Corporation, \\ 1-1 Hikarinooka, Yokosuka-shi, Kanagawa 239-0847, Japan \\ a)nakayama.yu@lab.ntt.co.jp
}

\begin{abstract}
Link metric optimization is crucial for load balancing in a shortest path bridging MAC (SPBM) network. Link metrics and traffic assignment proportions can be optimized by solving a linear programming problem using traffic demand. However, how to assign actual flows so that they are consistent with the optimized proportions has yet to be discussed. This paper proposes a rate-based path selection scheme. The concept is to assign flows to each path so that the total rates of the assigned flows approach the planned rates. With computer simulations, we confirmed that with the proposed scheme flows were assigned to each path so that they were consistent with the optimized proportions.
\end{abstract}

Keywords: SPBM, equal cost tree, multipath, link metric, load balance, fairness

Classification: Network

\section{References}

[1] Shortest Path Bridging, IEEE Standard 802.1aq, March 2012.

[2] Routing Bridges (RBridges): Base Protocol Specification, RFC6325, July 2011.

[3] OSI IS-IS Intra-domain Routing Protocol, RFC1142, February 1990.

[4] Y. Wang, Z. Wang, and L. Zhang, "Internet traffic engineering without full mesh overlaying," Proc. 20th Annual Joint Conference of the IEEE Computer and Communications Societies (INFOCOM 2001), pp. 565-571, April 2001. DOI:10. 1109/INFCOM.2001.916782

[5] Y. Nakayama and N. Oota, "Path selection algorithm for shortest path bridging in access networks," IEICE Commun. Express, vol. 2, no. 10, pp. 396-401, 2013. DOI:10.1587/comex.2.396

[6] Y. Nakayama, "Rate-based path selection for shortest path bridging in access networks," Proc. IEEE International Conference on Communications (ICC 2014), pp. 1272-1277, June 2014.

[7] Y. Nakayama and N. Oota, "Fairness with $\mathrm{n}$ rate $\mathrm{n}+1$ color marking on cascade aggregation for access network," Proc. IEEE Global Telecommunications Conference (GLOBECOM 2011), pp. 1-5, December 2011. DOI:10.1109/GLOCOM. 2011.6133675

[8] I. Stoica, S. Shenker, and H. Zhang, "Core-stateless fair queueing: a scalable architecture to approximate fair bandwidth allocations in high-speed networks," IEEE/ACM Trans. Netw., vol. 11, no. 1, pp. 33-46, February 2003. DOI:10.1109/ TNET.2002.808414 
[9] Visit http://www.isi.edu/nsnam/ns/ for Network Simulator ns-2.

\section{Introduction}

Server virtualization in data centers (DCs) has progressed significantly in recent years. Ethernet fabrics such as shortest path bridging (SPB) [1] and transparent interconnection of lots of links (TRILL) [2] have been standardized to realize the efficient use of network resources and a simple operating procedure. Ethernet fabrics employ the Intermediate System to Intermediate System (IS-IS) [3] protocol. By exchanging link states, shortest path transmission is realized without blocking ports.

Ethernet fabrics can be employed in access networks. Network operators can efficiently provide wide area Ethernet services with Ethernet fabrics. The high link utilization and simple operating procedure can be realized in any network topology. In particular, the use of SPB-MAC (SPBM) is expected to allow a smooth migration from existing networks because SPBM employs the provider backbone bridges (PBB) header as the outer MAC header. With SPBM, each backbone bridge (BB) advertises link states with IS-IS. The end-to-end shortest path is calculated to minimize the total link metrics along the path. If there are multiple shortest paths, each path is registered on the routing table as an equal cost tree (ECT). When a backbone edge bridge (BEB) receives a frame from outside the SPBM network, a path is selected from ECTs and the frame is transmitted by backbone core bridges (BCBs) along the selected path. Generally, this path allocation is performed statically with the frame header considering load balance.

Since the shortest paths are computed based on the link metrics, link metric optimization is crucial for load balancing over the entire network. The link metrics can be optimized by solving a linear programming problem using traffic demand [4]. This method determines the link metrics to minimize the maximum link utilization in the entire network. Consequently, it provides a path or a set of paths for each demand. If there are multiple paths for a demand, it also gives the proportions according to which the traffic should be distributed across them. However, how to assign actual flows so that they are consistent with the optimized proportions has yet to be discussed.

\section{Proposed scheme}

\subsection{Concept}

In this paper, we propose a rate-based path selection scheme based on a previously proposed load balancing scheme $[5,6]$. The concept of the proposed scheme is to assign flows to each path according to optimized proportions determined by the link metric optimization. Each BEB calculates the planned rate for each path using the demand and the optimized proportions. When a new flow arrives at a BEB, the BEB updates the estimated input rate of the flow and selects a path based on the planned rate and the rate distribution. The frame is transmitted to the path where the difference between the planned rate and the total input rate of the assigned flows is 
maximum. If the flow is continuous, the selected path is not updated to avoid frame reordering. Consequently, flows are assigned so that they are consistent with the optimized proportions.

\subsection{Path selection}

Let $i$ denote a flow identifier. Let $r_{i}$ and $p_{i}$ denote the estimated input rate and selected path for $i . t_{i}$ denotes the last frame arrival time of $i$. Let $j$ denote a path identifier for each path. $P_{j}$ denotes the planned rate for $j . R_{j}$ denotes the total rate of the assigned flows. Let $t$ denote the current time and $\delta$ denote a timeout value.

A BEB records the flow rate distribution in a flow table and a path table. In the flow table, the BEB records $r_{i}, t_{i}$, and $p_{i}$ for each flow from outside the SPBM network. The path table is generated for each set of ECTs and updated with the flow table. $P_{j}$ and $R_{j}$ for each path $j$ are recorded in the path table. The total rate $R_{j}$ is calculated as:

$$
R_{j}=\sum_{i} r_{i} \quad \text { s.t. } \quad p_{i}=j .
$$

The flow input rate can be estimated in many ways. The marking threshold of $\mathrm{N}$ rate $\mathrm{N}+1$ color marking $(\mathrm{NRN}+1 \mathrm{CM})$ [7] can be used. $d$ denotes the marking threshold and $w$ denotes the token accumulation rate. Since $d$ is incremented in accordance with the input rate, $(d+1) w$ can be used as the estimated input rate. With core-stateless fair queuing (CSFQ) [8], the input rate is estimated by using the frame arrival interval. Although the rate estimation methods can be employed independently, it is reasonable to employ them together with NRN+1CM or CSFQ for throughput fairness.

When a frame of $i$ arrives, the BEB determines the path along which to transmit the frame as:

1: update $r_{i}$

2: if $t-t_{i} \geq \delta$ then

3: $\quad R_{j} \Leftarrow \sum_{i} r_{i} \quad$ s.t. $\quad p_{i}=j$

4: $\quad p_{i} \Leftarrow j \quad$ s.t. $\quad \operatorname{Max}\left(P_{j}-R_{j}\right)$

5: end if

6: $t_{i} \Leftarrow t$

7: return $p_{i}$

After $r_{i}$ has been updated (line 1), the BEB decides whether $i$ is a new or continuous flow (line 2). If $t-t_{i} \geq \delta$ is satisfied, $i$ is considered a new flow. Otherwise, $i$ is considered a continuous flow. If $i$ is a new flow, $p_{i}$ is updated. $R_{j}$ is calculated as the total input rate of flows assigned to path $j$ (line 3 ). The path $j$ with the maximum difference between $P_{j}$ and $R_{j}$ is selected (line 4). Then, $t_{i}$ is updated (line 6).

If $i$ is a continuous flow, $p_{i}$ is not updated to avoid frame reordering. A major concern about splitting frames along multiple paths is frame reordering. Frame reordering can be avoided by setting the timeout value $\delta$ appropriately.

With this algorithm, the BEB selects the path based on the actual traffic distribution so that the total input rates approach the planned rate. Consequently, flows are assigned so that they are consistent with the optimized proportions. 


\section{Evaluation}

\subsection{Assumptions}

The performance of the proposed scheme is evaluated with simulations using network simulator ns-2 [9]. We confirmed the performance under a simple condition. The simulation topology is shown in Fig. 1. There were 4 bridges that were connected with SPBM. The link to the destination node was $1 \mathrm{Gbps}$ and other links were $100 \mathrm{Mbps}$. The link delay was $5 \mathrm{~ms}$ between BEBs and BCBs, and $0.05 \mathrm{~ms}$ for the other links. The queue length was $1 \mathrm{MB}$ for all bridges.

120 user nodes were linked to BEB0. The user nodes send flows to the destination node. The total traffic demand was assumed to be $200 \mathrm{Mbps}$. In this case, the optimal link metrics were 1 for all links. There are multiple shortest paths for the user flows. The optimized proportions of the traffic allocation were 0.5 and 0.5 for the paths via BCB 0 and $\mathrm{BCB} 1$. Therefore, the planned rates were $100 \mathrm{Mbps}$ for each path. We assumed that there was a bias in the flow rate distribution. 100 user nodes send UDP flows at an average rate of $1 \mathrm{Mbps}$ and 20 user nodes send UDP flows at an average rate of $5 \mathrm{Mbps}$. The simulation time was $20 \mathrm{~s}$. The frame length was $1.5 \mathrm{~KB}$. To simplify the simulation, the PBB header length was not considered. $\delta$ was set at $30 \mathrm{~ms}$. To realize per-flow throughput fairness during congestion, NRN+1CM [7] was employed in the SPBM. The parameters in [7] were set at $N+1=16, w=0.5 \mathrm{Mbps}$, and token bucket length $B=2.5 \mathrm{~KB}$.

The flow throughput was measured using the above conditions. The proposed scheme was compared with a simple distribution method that assigns flows so that the numbers of flows assigned to each path were the same.

\subsection{Result}

Since the total traffic demand was $200 \mathrm{Mbps}$ and the traffic assignment proportions were 0.5 , the planned rates were $100 \mathrm{Mbps}$ for each flow.

Fig. 2 shows the total rate of the assigned flows for each path. Fig. 3 shows the throughput histogram of each flow. With the proposed scheme, the total rates were consistent with the planned rates for both paths. The flow throughputs were almost the same as their output rates at the source nodes, because a small number of frames were dropped. The total throughput of all the flows was $185 \mathrm{Mbps}$. With the simple distribution method, the rate distribution depends on the traffic rate bias of the assigned flows. Fig. $2 \mathrm{~b}$ shows the worst case. Although the path via BCB0 was $100 \%$ used, the path via BCB1 was underutilized. The flow throughputs decreased from their output rates at the source nodes, because frames were discarded on the congested path. The total throughput of all flows decreased to $161 \mathrm{Mbps}$. This result implies that if there is a bias in the flow rate distribution, it is difficult to achieve the planned rate with a simple flow assignment.

Therefore, the proposed scheme can select a path in accordance with the planned rate and realize good load balancing. 


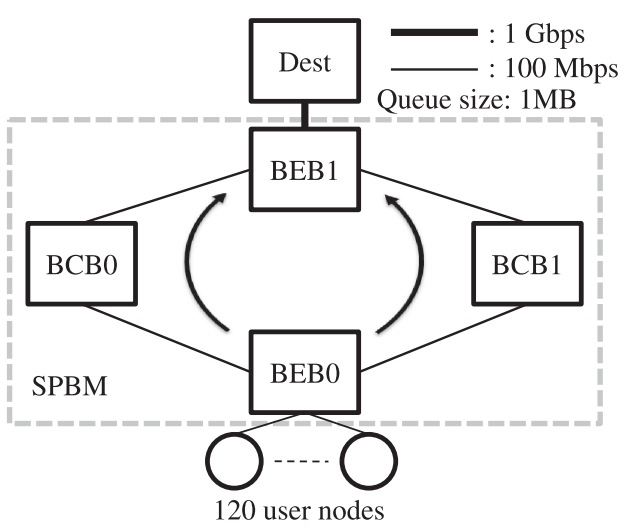

Fig. 1. Simulation topology.

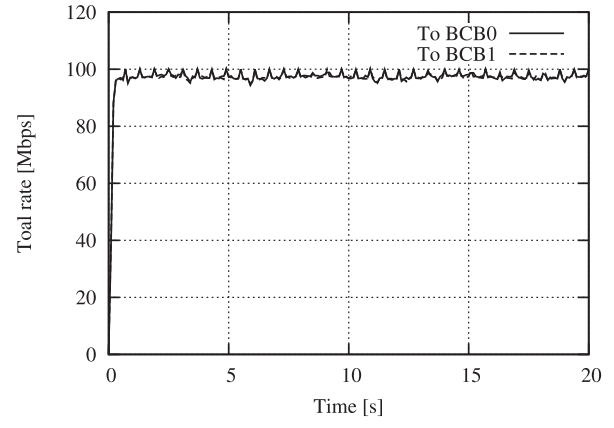

(a) Proposed scheme

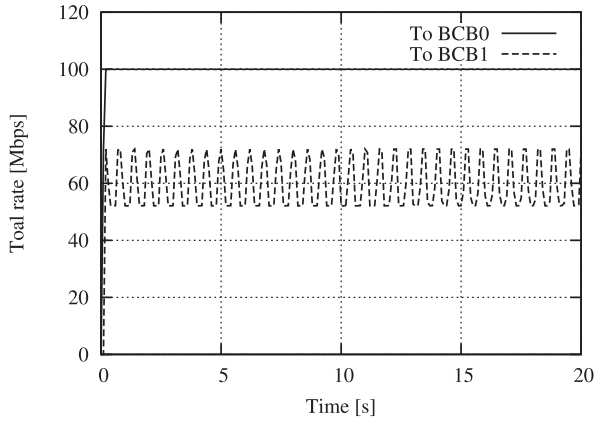

(b) Hash function

Fig. 2. Total rate of each path.

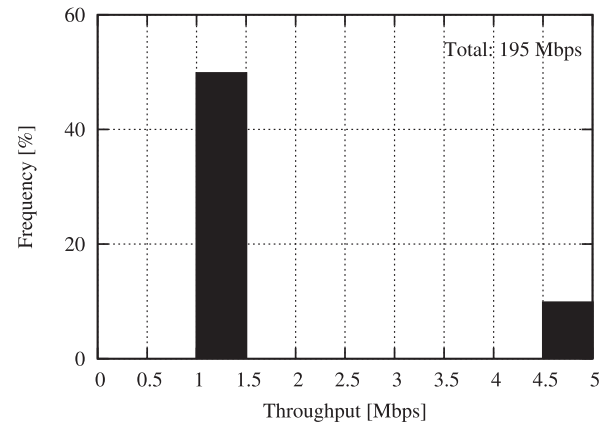

(a) Proposed scheme

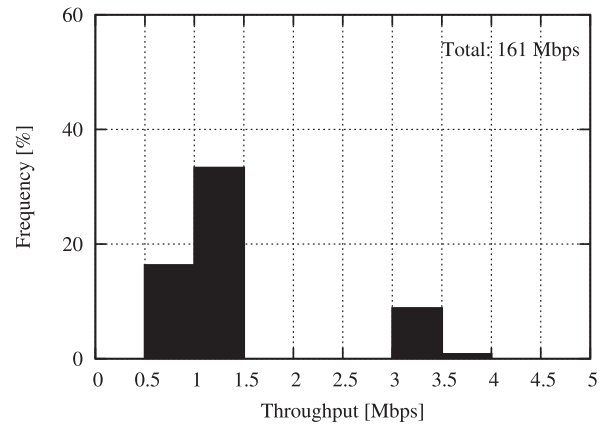

(b) Hash function

Fig. 3. Flow throughput.

\section{Conclusion}

This paper proposed a rate-based path selection scheme. The concept of the proposed scheme is to assign flows to each path according to optimized proportions determined by link metric optimization. Each bridge calculates the planned rate for each path using the demand and the optimized proportions. When a new flow arrives, a path is selected so that the total rates of the assigned flows approach the planned rates. We confirmed using computer simulations that with the proposed scheme flows are assigned to each path so that they are consistent with the optimized proportions. 